\title{
Snake Venom Metalloproteinases
}

\author{
Gâz Florea Șerban Andrei ${ }^{1 *}$, Gâz Florea Adriana², Kelemen Hajnal ${ }^{3}$ Daniela-Lucia Muntean \\ 1 Fundamental Pharmaceutical Science Department, Faculty of Pharmacy, University of Medicine and Pharmacy, Tîrgu Mureș, Romania \\ 2 Mureș Water Administration, Tîrgu Mureș, Romania \\ ${ }^{3}$ Specialist Pharmaceutical Science Department, Faculty of Pharmacy, University of Medicine and Pharmacy, Tîrgu Mureș, Romania
}

As more data are generated from proteome and transcriptome analysis revealing that metalloproteinases represent most of the Viperid and Colubrid venom components authors decided to describe in a short review a classification and some of the multiple activities of snake venom metalloproteinases. SVMPs are classified in three major classes (P-I, P-II and P-III classes) based on the presence of various domain structures and according to their domain organization. Furthermore, P-II and P-III classes were separated in subclasses based on distinctive post-translational modifications. SVMPs are synthesized in a latent form, being activated through a Cys-switch mechanism similar to matrix metalloproteinases. Most of the metalloproteinases of the snake venom are responsible for the hemorrhagic events but also have fibrinogenoIytic activity, poses apoptotic activity, activate blood coagulation factor II and X, inhibit platelet aggregation, demonstrating that SVMPs have multiple functions in addition to well-known hemorrhagic function.

Keywords: snake venom metalloproteinases, hemorrhagic activity, hemostatic distruption, ADAM and ADAMTS, SVMPs

Received: 18 August 2015 / Accepted: 21 September 2015

\section{Introduction}

Snakes have fascinated humankind for millennia, being worshiped in antiquity by almost all the civilizations. In Ancient Egypt a Cobra adorned the pharaoh's crown, in Greece snakes could be found in many medical symbols, while in India snakes even had their own festival (Nag Panchami) [1,2].The destructive effect of snake venom on living organism was well known, but their healing potential has only been considered in the last 2 centuries. Snake venoms are typically a complex cocktail of mostly peptides, proteins, enzymes and other small substances with toxic and lethal properties that facilitate the immobilization and digestion of the prey, as well as providing a defense against predators. The life or death of these prey/predator encounters forced the toxins to be fast-acting and potent molecules. The most common snake venom enzymes include; cysteine-rich secretory proteins (CRiSPs), three-finger toxins (3FTXs) especially in Elapidae family, phospholipase $\mathrm{A}_{2}$, metalloproteinases, L-amino acid oxidase, serine proteinases, acetylcholinesterase, and phosphodiesterase [3].

\section{History}

Early studies by Reichert and Mitchell noticed that fibrinogen in animal blood loses its ability to coagulate after exposure to snake venom. They observed that when animal blood was mixed with Crotalus family venom, the blood clot that was formed was dissolved within 24 hours while sitting at room temperature $[4,5]$. Probably the first report of SVMPs was by Ohsaka, Okonogi and Maeno when they observed that isolated proteinases toxin from viperid were inactivated by exposure to EDTA, presumably because of the presence of a metal inside the protein, most likely being a metalloproteinase [6-9].

* Correspondence to: Șerban Andrei Gâz Florea

E-mail: andrei.gaz@umftgm.ro
Since the discovery of zinc-dependent proteinase in the viperid venom [10], researchers have tried to isolate, characterize and understand the structure and biological function in order to find their role in the envenomation of the prey [11]. Proteinase represents one of the enzymes found in the venom of many snakes, and are structurally classified as serine proteinases and metalloproteinases [12]. The most intriguing enzyme is metalloproteinase and it is estimated that it is at least $30 \%$ of the total protein content of most viperid venoms [13]. Soon after the discovery of the snake venom metalloproteinases and the first sequence determination of them, it was thought that they are part of the matrix metalloproteinases family [14]. However, Bjarnason and Fox discovered that snake venom metalloproteinases were part of a new family of metalloproteinases, distinct from matrix metalloproteinase $[15,16]$.

\section{Classification}

As major classes, proteinase can be classified in exopeptidase (e.g. aminopeptidase, cathepsin A) and endopeptidase (ADAMs and matrix metalloproteianse). ADAMs stands

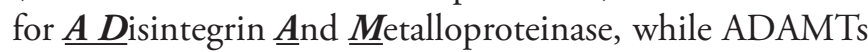
are $\underline{\boldsymbol{A} D}$ isintegrin $\underline{\boldsymbol{A}}$ nd $\underline{\boldsymbol{M}}$ etalloproteinase with $\underline{T}$ hrombospondin motifs.

SVMPs were originally classified into structural classes based on the presence of various domain structures and according to their domain organization [15]. All SVMPs share a metalloproteinase domain where the metallic ion is either zinc (most of the metalloproteinases from the snake venom) or cobalt, characterized by the HEXXHXXGXXH metal (zinc)-binding motif. Being an extracellular protein, it is expected to have structurally stabilizing disulfide bonds. cDNA sequence analysis indicate that SVMPs are synthesized in a latent form, being 
activated through a Cysteine-switch mechanism similar to matrix metalloproteinases $[17,18]$.

Schematically, snake venom metalloproteinases can be classified in three major classes (figure 1), originally developed by Fox and Serrano in 2005 [19]. Usually ADAMs are multi-domain proteins that are minimally comprised of metalloproteinase and disintegrin-like domains. In addition, a peptide signaling sequence can be found in the beginning of the protein chain. Some ADAMs also display additional domains, such as transmembrane and cytoplasmic, as well as thrombospondin domains [20].

The first class, named P-I is the simplest SVMPs, comprised of only a metalloproteinase domain. In their nascent form, they have a peptide signal sequence, a pre-pro-domain, which undergoproteolytically in the mature SVMPs, followed by a "spacer" domain towards with carboxy group to metalloproteinase domain. The molecular weight of these classes of metalloproteinaseslies around $25 \mathrm{kDa}$, with 2 sub-domains, a major one ( $4 \alpha$-helix and $\beta$-folding) and a minor (with only one $\alpha$-helix). Usually P-I class has 4 to 6 cysteine residues in the protein.

The second class of SVMPs is P-II, which is somewhat similar to P-I class, being characterized by the presence of a disintegrin domain in the nascent form, which in most cases hosts the canonical RGD (arginine-glycine-aspartic acid) motif. The P-II class was subdivided in 5 different subclasses (P-IIa, P-IIb, P-IIc, P-IId and P-IIe) of metalloproteinases. The difference between all subclasses arise on the disintegrin domain based on their distinctive posttranslational modifications.

The disintegrin domain is found carboxy to the spacer domain, which can be modified proteolytically to a "free" disintegrin. The molecular weight of P-II classes is between 25 and $50 \mathrm{kDa}$.

In P-IIb disintegrin domain is not processed, remaining part of the proteinase, while P-IIc is in fact a dimeric form of P-IIb. P-IId is a precursor from which a homodimeric disintegrin found in venoms is formed, while P-Ile represents the actual P-II class of snake venom metalloproteinases, which give rise to a heterodimeric RGD-containing disintegrin [21].

The P-III SVMPs family has much more domains than P-II class, bearing signaling peptide sequence, pro-domain, metalloproteinase, spacer, disintegrin-like and cysteinerich domains (figure 2) in the nascent forms, carboxy to the spacer domain. P-III family is divided in 4 subclasses having differences along the disintegrin-like and cysteinerich domains.

It is one of the heaviest family of metalloproteinases often having molecular weight of more than $50 \mathrm{kDa}$. Even

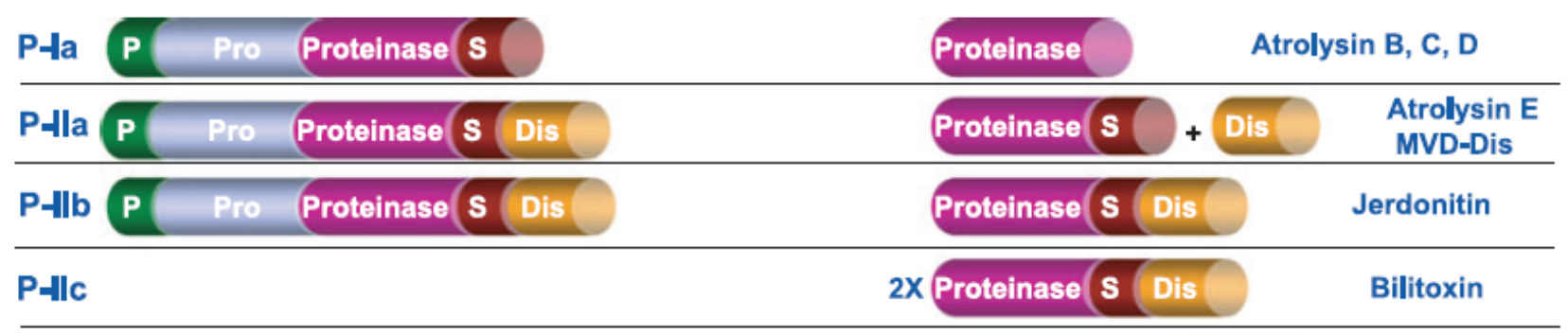

P-lld P Pro Proteinase $\mathbf{S}$ Dis

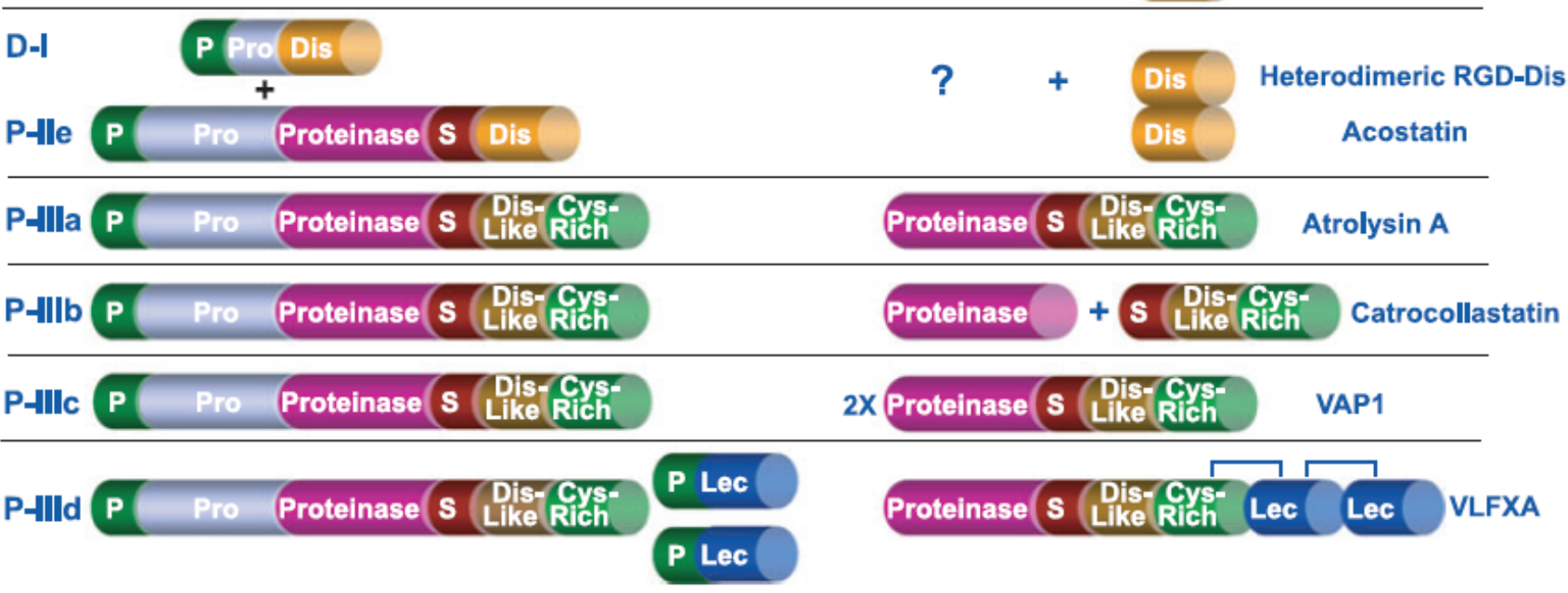

Fig. 1. Schematic of snake venom metalloproteinases classes (reprinted from Fox and Serrano, 2008 [13], with permission from Wiley). Question marks (?) from the figure indicate the lack of processed product identification in the venom. In the figure, P means signaling peptide sequence, Pro means a pro-domain, S stands for "spacer", Dis is disintegrin or Dis-Like a disintegrin-like domain, Cys-Rich represents a cysteine-rich domain and Lec is lectin domain. 


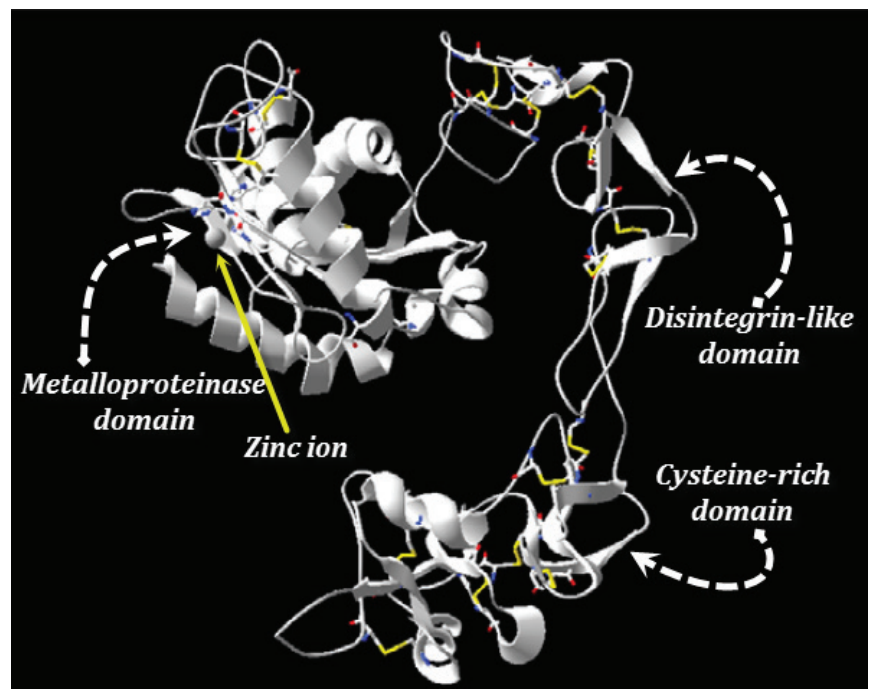

Fig. 2. Structural analysis of the P-III SVMP jararhagin model (reprinted from Pinto and Fox, 2007 [22], from Elsevier)

if disintegrin-like domain shares some sequence homology with the disintegrin domain of the P-II family, they are distinct, having different disulfide bond structure.

P-IIIa is somewhat similar to P-IIb, where disintegrinlike and cysteine-rich domains do not undergo any posttranslational modifications. In contrast, in the P-IIIb subclass, the spacer, the disintegrin-like and cysteine-rich domains are proteolytically modified from the metalloproteinase domain [21]. Up to date, there is uncertainty about the processing mechanism for the metalloproteinase domain of the spacer, disintegrin-like and cysteine-rich domains. Moura and coworkers, however, suggested that the processing of the domains from the proteinase is linked with the disulfide bond arrangement and/or folding of the protein [23].

P-IIIc is practically the dimeric form of P-IIIa, while P-IIId subclass is a modified P-IIIa structure with the addition of two disulfide-bonded lectin-like domains posttranslationally coupled to the P-IIIa structure.

\section{Proteomic analysis of snake venom for metalloproteinases and SVMPs structure}

Recent proteomic analyses of snake venom have shown that SVMPs are the major components of venom of $\mathrm{Vi-}$ peridae family, varying from roughly $11 \%$ up to almost 65\% [24]. But SVMPs are ubiquitously distributed in almost all venomous snake species being present in Colubridae family $[25,26]$ as well as in the Elapidae (in some members) family $[27,28]$ although in much lower quantity than in Viperidae family.

Practically almost all of the SVMPs are zinc-dependent but cobalt can be also involved. The metal inside the metalloproteinase is tetra coordinated, three ligands being coordinated from protein (usually histidine, lysine, arginine etc.) and one with a labile water molecule.

Inactivation of snake venom metalloproteinases can be done using chelating agent such as ethylenediaminetetraacetic acid (EDTA) or o-phenanthroline [21].
SVMPs are synthetized in cytoplasm of specialized secretory glands cells and transferred to the rough endoplasmic reticulum, then to the Golgi apparatus, and finally transported to the lumen of the venom gland viasecretory granules.

Studying cDNA Fox and Serrano observed that every metalloproteinases from the snake venom have a signaling sequence that probably targets a nascent protein to a signal recognition site or protein on the endoplasmic reticulum. The peptide signal sequence removing occur during transport into to endoplasmic reticulum (ER). It has also been presumed that folding of the protein takes place in the rough ER and undergo disulfide bond oxidation/formation, glycosylation or dimerization/polymerization of the nascent proteins [13].

Incorrectly, folded proteins, which takes place in the venom gland secretory, will not be transferred to the Golgi network. Fox suggested that in the trans-Golgi network nascent preform of the proteins would be proteolytically processed [13], as it happens with most of the latent proteins. The process is most probably completed at the time that the vesicles are released into the gland lumen (figure 3).

Shimokawa and collaborators have analyzed a recombinant pro-atrolysin E (a P-IIa SVMP from Crotalus atrox) and they have noticed, "in vitro" an autolytic processing which occur via an initial proteolytic cleavage to release the pro-domain followed by a second cleavage to the disintegrin domain [18]. They also noticed that SVMPs have a functional Cysteine-switch mechanism for maintaining the protein in a latent form. Analyzing a P-IIIb class SVMP jararhagin from Bothrops jararaca Shimokawa [28] and Moura-da-Silva [23] observed that this class undergoes to a post-translation process where a disintegrin-like/cysteinerich domain are released giving rise to a jararhagin-C, but not on entire population of the proteins. There are still part of the proteins population that do not undergo on this post-processing, one possible explanations could be that there are different folding isomers and they can be differentially processed in order to release disintegrin-like/ cysteine-rich domain, explaining the snake venom complexity and function.

Another important characteristic of SVMPs structure are disulfide bonds. Usually a P-I subclass has two to three disulfide bonds in the metalloproteinase domain. In the P-II and P-III subclasses that have often an odd number of cysteinyl residues there are slightly more disulfide-bonds inside the metalloproteinase domain. In the P-II and P-III subclasses cysteinyl residue can be found as free cysteine or form disulfide bonds not only inside a specific domain but also with cysteinyl residues from other domains, or forming dimeric or polymeric structures.

P-II subclass usually exhibit about 5 to 7 disulfide bonds inside the disintegrin domains, while P-III subclass has around 8 disulfide bonds in the disintegrin-like domain, 6 in the cysteine-rich domain and 3 disulfide bonds in the lectin-like domain of P-IIId. 
IV. Golgi

III. ER

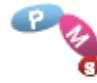

II. ER

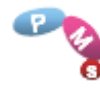

ER

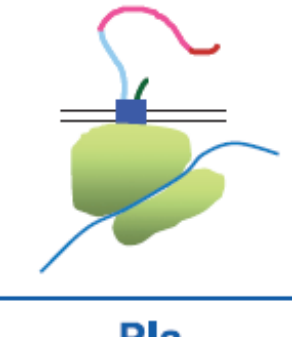

$\mathrm{Pla}$
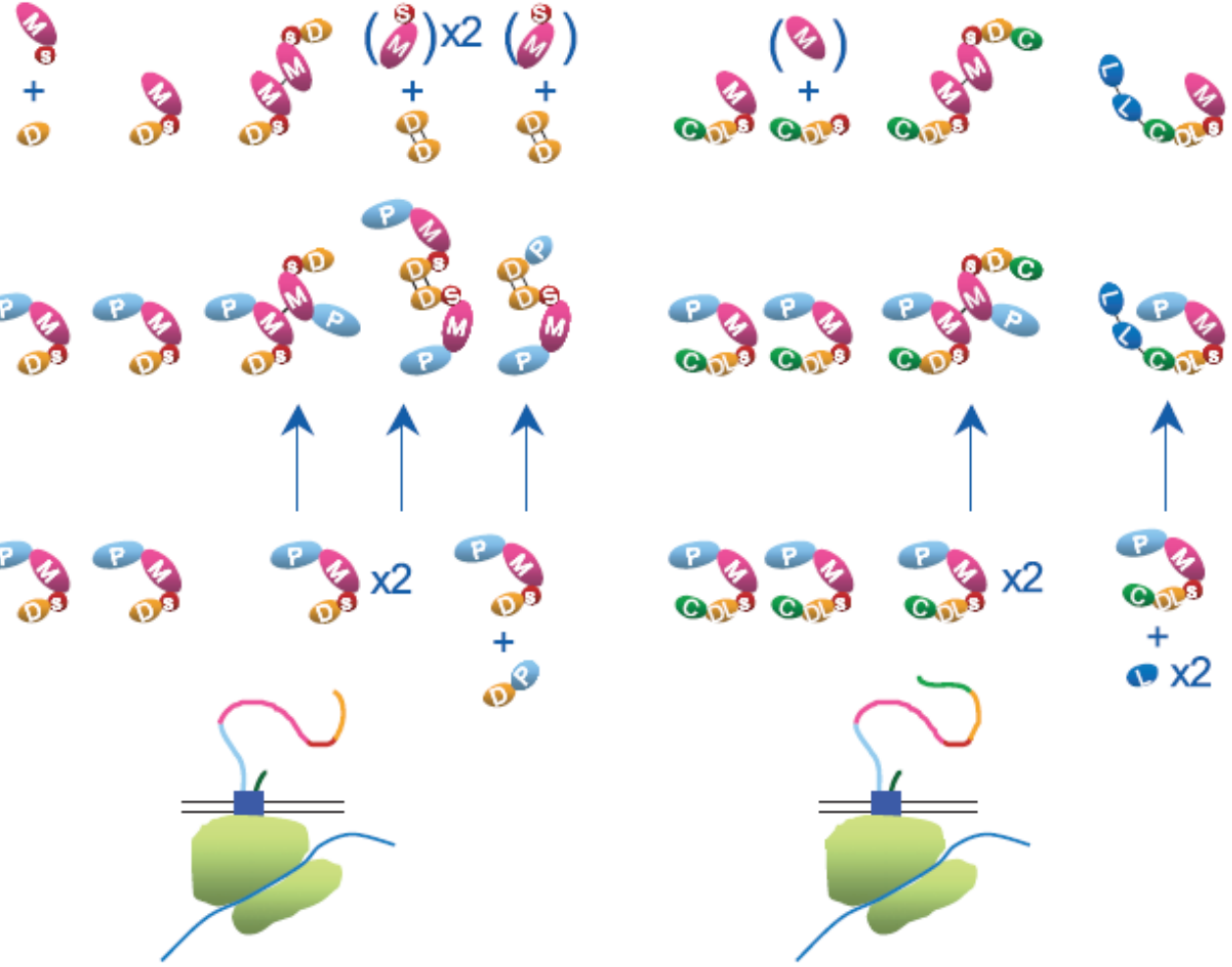

Plla, PIlb, Pllc, PIld, Plle

PIIla, PIIlb, PIIIc, PIIId

Fig. 3. Schematic representing a hypothetical biosynthesis pathway from transcription on the ER surface, through endoplasmic reticulum to the Golgi network and release of the secretory vesicles into the venom lumen for the production of the three SVMPs venom classes. In the figure, $\mathrm{P}$ means a pro-domain, $\mathrm{M}$ is metalloproteinase domain, S stands for "spacer", D is disintegrin or DL a disintegrin-like domain, Cys represents a cysteine-rich domain and L is lectin-like domain (reprinted from Fox and Serrano, 2008 [13], with permission from Wiley).

\section{Function of SVMPs}

Wide varieties of biochemical and biological activities have been described to the SVMP, showing therefore their critical role in the overall toxicity of the venom. SVMPs are responsible for the local and systemic hemorrhagic activity, myonecrosis [17], disruption of hemostasis mediated by procoagulant or anticoagulant effects, platelet aggregation, necrosis or pro-inflamatory activities. Therefore, the most important role of metalloproteinases seems to be in the prey predigestion. Table I summarize some of the activities of some metalloproteinases isolated from snake venom.

Table I exhibits a brief summary of some of the biological activities associated with some SVMPs. As it can be seen most of the functional activities of SVMPs are associated with hemorrhagic or the disruption of the hemostatic system. All these activities are mediated by the proteolytic activity of metalloproteinase domain of SNVMs. Blood coagulation proteins (e.g. fibrinogen, factor $\mathrm{X}$ and factor II, prothrombin) are also targets of their proteolytic activities showing fibrinogenolytic activity, inhibition of platelet aggregation and activation of factor II and X.

\section{Conclusions}

Since SVMPs represent an important and major class of snake venom proteins as revealed by proteomic analysis and many of these proteinase have biological activity we have collected new data, especially after 2000 , in a mini- review. In this mini-review, we intended to present, based on literature data, the current understanding of the structural features that contribute to complexity of snake venom metalloproteinases. There is described classification of SVMPs as well as biological function of them. Most of the metalloproteinases of the snake venom are responsible for the hemorrhagic events that occur in the case of envenomation therefore represents a significant health hazard for the victims. We believed the venoms of snake could represent a huge and important source for potent pharmacological active molecules or for lead molecules that should be explores as much as possible.

\section{Acknowledgments}

This paper was published under the frame of European Social Found, Human Resources Development Operational Programme 2007-2013, project no. POSDRU/159/1.5/S/136893.

We would like to thanks to Michael Holmes (Canada) for a brief review, drafting and editing the article in English.

\section{References}

1. http://www.outsideonline.com/1912971/venom-not-just-poison

2. http://www.wondersandmarvels.com/2011/11/the-uses-of-snakevenom-in-antiquity.htm

3. Kang TS, Georgieva D, Kini RM et al. Enzymatic toxins from snake venom: structural characterization and mechanism of catalysis. FEBS J. 
Table I. Biological activities of selected SVMPs

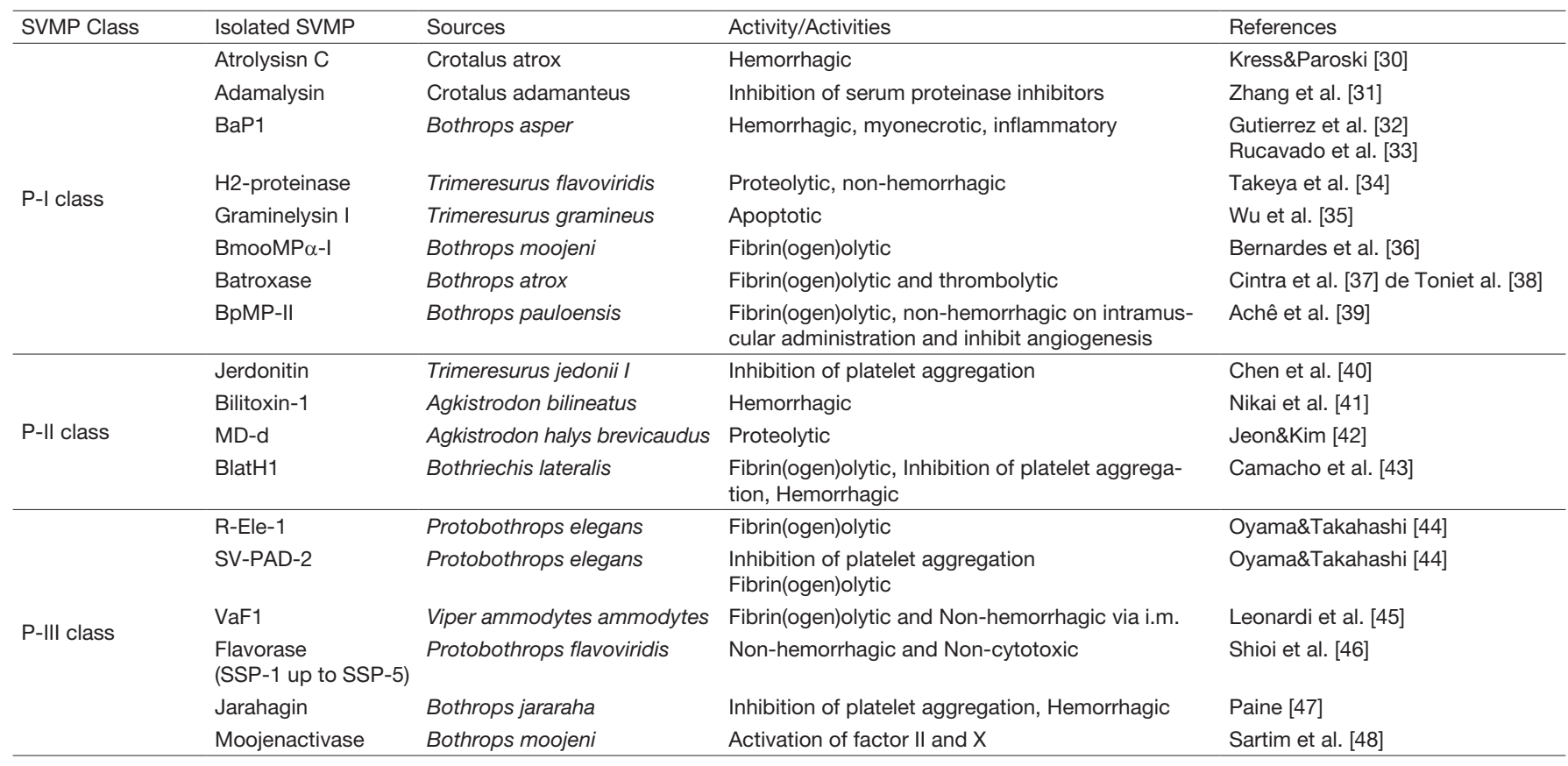

2011;278(23):4544-4476.

4. Mitchell SW, Reichert ET. Researches upon the venoms of poisonous serpents.Smithsonian Contribution to Knowledge Smithsonian Institute, Washington D.C. 1886;pp.89-95.

5. Markland Jr. FS, Swenson S. Snake venom metalloproteinases. Toxicon. 2013;62:3-18.

6. Ohsaka A. Fractionation of Habu snake venom by chromatography on $\mathrm{cm}$-cellulose with special reference to biological activities. Jpn J Med Sci Biol. 1960;13:199-205

7. Onsaka A, Ikezawa $\mathrm{H}$, Kondo $\mathrm{H}$, Kondo S. Two hemorrhagic principles derived from Habu snake venom and their difference in zone electrophoretical mobility. Jpn J Med Sci Biol. 1960;13:73-76.

8. Okonogi T, Hoshi S, Honma M, et al. Studies on the habu snake venom. 3-2. A comparative study of histopathological changes caused by crude venom, purified habu-proteinase and other proteinases. Jpn J Microbiol. 1960;4:189-192.

9. Maeno H, Mitsuhashi S, Sato R. Studies on Habu snake venom. 2c. Studies on Hß-proteinase of Habu venom.Jpn J Microbiol. 1960;4:173180.

10. Bjarnason JB, Tu AT. Hemorrhagic toxins from western diamondback rattlesnake (Crotalus atrox) venom: isolation and characterization of five toxins and the role of zinc in hemorrhagic toxin e. Biochemistry. 1978;17(16):3395-3404.

11. Takahashi T, Onsaka A. Purification and some properties of two hemorrhagic principles (HR2a and HR2b) in the venom of Trimeresurus flavoviridis; complete separation of the principles from proteolytic activity. Biochim Biophys Acta. 1970;207(1):65-75.

12. Takeda S, Takeya H, Iwanaga S. Snake venom metalloproteinase: structure, function and relevance to the mammalian ADAM/ADAMTS family proteins. Biochim Biophys Acta. 2012;1824(1):164-176.

13. Fox JW, Serrano SMT. Insights into and speculations about snake venom metalloproteinase (SVMP) synthesis, folding and disulfide bond formation and their contribution to venom complexity. FEBS J. 2008;275:30163030.

14. Shannon JD, Baramovat EN, Bjarnason JB, Fox JW. Amino acid sequence of a Crotalus atrox venom metalloproteinase which cleaves type IV collagen and gelatin. J Biol Chem. 1989;264(20):11575-11583.

15. Bjarnason JB, Fox JW. Snake venom metalloendopeptidases: Reprolysins. Methods Enzymol. 1995;248:345-368.

16. Bjarnason JB, Fox JW. Hemorrhagic metalloproteinases from snake venoms. Pharmacol Ther. 1994;62(3):325-372.

17. Hite LA, Shannon JD, Bjarnason JB, Fox JW. Sequence of cDNA clone encoding the zinc metalloproteinase hemorrhagic toxin e from Crotalus atrox: Evidence for signal, zymogen, and disintegrin-like structures. Biochemistry. 1992;31:6203-6211.

18. Shimokawa K, Jia LG, Wang XM, Fox JW. Expression, activation, and processing of the recombinant snake venom metalloproteinase, proatrolysin E. Arch BiochemBiophys. 1996;335(2):283-294.
19. Fox JW, Serano SMT. Structural considerations of the snake venom metalloproteinases, key members of the M12 reprolysin family of metalloproteinases. Toxicon. 2005; 45: 969-985;

20. White JM. ADAMs: modulators of cell-cell and cell-matrix interactions. CurrOpin Cell Biol. 2003;15(5):598-606.

21. Mackessy S. Handbook of Venom and Toxins of Reptiles. CRC Press, Taylor \& Francis Group, Boca Raton, Florida, USA; 2010;P.12-16,P. 95132.

22. Pinto A FM, Terra MS, Guimares JA, Fox JW. Mapping von Willebrand factor A domain binding sites on a snake venom metalloproteinase cysteine-rich domain. Arch Biochem Biophys. 2007;457(1):41-46.

23. Moura-da-Silva AM, Della-Casa MS, David AS, Assakura MT, Butera D, Lebrun I, Shannon JD, Serrano SM, Fox JW. Evidence for heterogeneous forms of the snake venom metalloproteinase jararhagin: A factor contributing to snake venom variability. Arch BiochemBiophys. 2003;409(2):395-401.

24. Calvete JJ, Juarez P, Sanz L. Snake venomics. Strategy and applications.J Mass Spectrom. 2007;42:1405-1414.

25. Ompraba C, Chapeaurouge A, Kini RM et al. Identification of a novel family of snake venom proteins veficolins from Cerberus rynchops using a venom gland transcriptomics and proteomics approach. J Proteome Res. 2010;9(4):1882-1893.

26. Weldon CL, Mackessy SP. Biological and proteomic analysis of venom from the Puerto Rican Racer (Alsophis Portoricensis: Dipsadidae). Toxicon. 2010;55:558-569.

27. Georgieva D, Seifert J, Betzel C et al. Pseudechis australis venomics: adaptation for a defense against microbial pathogens and recruitment of body transferrin. 2011;10(5):2440-2464.

28. Petras D, Sanz L, Calvette JJ et al. Snake venomics of African spitting cobras: toxin composition and assessment of congeneric cross-reactivity of the pan-African EchiTAb-Plus-ICP antivenom by antivenomics and neutralization approaches. J. ProteomeRes. 2011;10:1266-1280.

29. Shimokawa K, Shannon JD, Jia LG, Fox JW. Sequence and biological activity of Catrocollastatin-C: a disintegrin-like/cysteine-rich twodomain protein from Crotalus atrox venom. Arch BiochemBiophys. 1997;343(1):35-43.

30. Kress LF, Paroski EA. Enzymatic inactivation of human serum proteinase inhibitors by snake venom proteinase. BiochemBiophys Res Commun. 1978;83:649-656.

31. Zhang D, Fox JW, Meyer EF et al. Structural interaction of natural and synthetic inhibitors with the venom metalloproteinase atrolysin $\mathrm{C}$ (form d). ProcNatlAcadSci USA 1994;91(18):8447-8451.

32. Gutierrez JM, Romero M, Diaz C, Borkow G, Ovadia M. Isolation and characterization of a metalloproteinase with weak hemorrhagic activity from the venom of the snake Bothrops asper (terciopelo). Toxicon. 1995;33:19-29.

33. Rucavado A, Lomonte B, Ovadia M, Gutierrez JM. Local tissue damage induced by BaP1, a metalloproteinase isolated from Bothrops asper 
(terciopelo) snake venom. ExpMolPathol. 1995;63:186-199.

34. Takeya H, Arakawa M, Miyata T, Iwanaga S, Omori-Satoh T. Primary structure of $\mathrm{H} 2$-proteinase, a non-hemorrhagic metalloproteinase, isolated from the venom of the habu snake, Trimeresurus flavoviridis. $J$ Biochem. 1989;106:151-157.

35. Wu WB, Chang SC, Liau MY, Huang TF. Purification, molecular cloning and mechanism of action of graminelysin I, a snake-venom-derived metalloproteinase that induces apoptosis of human endothelial cells. Biochem J. 2001;357:719-728.

36. Bernardes CP, Soares AM, de Oliveira F et al. Isolation and structural characterization of a new fibrin(ogen)olytic metalloproteinase from Bothrops moojeni snake venom. Toxicon. 2008;51:574-584.

37. Cintra AC, De Toni LG, Sampaio SV et al. Batroxase, a new metalloproteinase from $B$. atrox snake venom with strong fibrinolytic activity. Toxicon. 2012;60(1):70-82.

38. de Toni LGB, Menaldo DL, Sampaio SV. Inflammatory mediators involved in the paw edema and hyperalgesia induced by Batroxase, a metalloproteinase isolated from Bothrops atrox snake venom. Int Immunopharmacol. 2015;28(1):199-207.

39. Achê DC, Gomes MS, Rodrigues VdeM et al. Biochemical properties of a new PI SVMP from Bothrops pauloensis: inhibition of cell adhesion and angiogenesis. Int J Biol Macromol. 2015;72:445-453.

40. Chen $R Q$, Jin $Y$, Xiong $Y L$ et al. A new protein structure of $P$-II class snake venom metalloproteinase: it comprise metalloproteinase and disintegrin domains. Biochem Biophys Res Commun. 2003;310:182-187.

41. Nikai T, Fox JW, Sugihara $H$ et al. Primary structure and functional characterization of bilitoxin-1, a novel dimeric P-\|l snake venom metalloproteinase from Agkistrodon bilineatus venom. Arch Biochem
Biophys. 2000;378:6-15.

42. Jeon $\mathrm{OH}$, Kim DS. Molecular cloning and functional characterization of a snake venom metalloproteinase.Eur J Biochem. 1999;263:526-533.

43. Camacho E, Gutierrez JM, Rucavado A et al. Understanding structural and functional aspects of Pll snake venom metalloproteinase: Characterization of BlatH1, a hemorrhagic dimeric enzyme from the venom of Bothriechis lateralis. Biochimie. 2014;101:145-155.

44. Oyama E, Takahashi H. Purification and characterization of two high molecular mass snake venom metalloproteinase (P-III SVMPs), named SV-PAD-2 and HR-Ele-1, from the venom of Protobothrops elegansi (Sakishima-habu). Toxicon. 2015;103:30-38.

45. Leonardi A, Sajevic T, Križaj I et al. Structural and biochemical characterization of VaF1, a P-Illafibrinogenolytic metalloproteinase from Vipera ammodytes ammodytes venom. Biochimie. 2015;109:78-87.

46. Shioi N, Nishijima A, Terada S. Flavorase, a novel non-hemorrhagic metalloproteinase in Protobothrops flavoviridis venom, is a target molecule of small serum protein-3. J Biochem. 2015;158(1):37-48.

47. Paine MJ, Desmond HP, Theakston RD, Crampton JM. Purification, cloning and molecular characterization of high molecular weight hemorrhagic metalloproteinase, jarahagin from Bothrops jararaca venom. Insights into the disintegrin gene family. J Biol Chem. 1992;267:2286922876.

48. Sartim MA, Costa TR, Sampaio SV et al. Moojenactivase, a novel procoagulant Pllldmetalloprotease isolated from Bothrops moojeni snake venom, activates coagulation factors $I I$ and $X$ and induces tissue factor up-regulation in leukocytes. Arch Toxicol. 2015;DOl10.1007/s00204015-1533-6. 